\title{
As ações de enfermagem voltadas a permanência do paciente esquizofrênico vinculado ao Centro de Atenção Psicossocial CAPS
}

\author{
Nursing Actions Focused on Patient Permanence Schizophrenic Linked to the Psychosocial Care \\ Center CAPS \\ Acciones de Enfermería Orientadas a la Permanencia del Paciente Esquizofrénico Vinculado al
}

\author{
Centro de Atención Psicosocial CAPS
}

Recebido: 03/04/2021 | Revisado: 09/05/2021 | Aceito: 09/05/2021 | Publicado: 18/05/2021

\author{
Lara Priscila Lemos Leite \\ ORCID: https://orcid.org/0000-0002-8657-9476 \\ Centro Universitário Santo Agostinho, Brasil \\ E-mail:lemoslara58@gmail.com \\ Karine Rodrigues dos Santos \\ ORCID: https://orcid.org/0000-0002-7583-0717 \\ Centro Universitário Santo Agostinho, Brasil \\ E-mail:karinecoelho22@gmail.com \\ Laurimary Caminha Veloso \\ ORCID: https://orcid.org/0000-0002-3245-6307 \\ Centro Universitário Santo Agostinho, Brasil \\ E-mail:laurimary.caminha@gmail.com
}

\begin{abstract}
Resumo
A esquizofrenia é um transtorno psiquiátrico complexo de inicio cedo na vida do indivíduo e que apresenta diversos déficits sociais e cognitivos caracterizados por distorções do pensamento, da percepção de si, delírios, alucinações, psicose e da realidade externa. Objetivou-se analisar as ações desenvolvidas pelos enfermeiros nos Centros de Atenção Psicossocial CAPS voltados para a permanência do paciente esquizofrênico no tratamento. Realizou-se um estudo de revisão integrativa da literatura com várias buscas nas bases de dados Biblioteca Virtual em Saúde (BVS), BDENF, LILACS, SCIELO, com os descritores: "Enfermeiro", "Esquizofrenia" e "CAPS". Foram selecionados ao todo 305 artigos, que logo após foram aplicados os critérios de inclusão e exclusão e foram selecionados 31 artigos para leitura. Após a leitura foram selecionados 07 artigos que atendiam ao objetivo da pesquisa. Os resultados da amostra evidenciaram que as ações já existentes estão em constante aprimoração e desenvolvendo -se novas estratégias para que haja a permanência do paciente no tratamento. Conclui-se que o enfermeiro se destaca no cuidado prestado ao usuário com esquizofrenia nos serviços de saúde, devido o vínculo estabelecido e responsabilidade na estruturação de estratégias de promoção em saúde mental.
\end{abstract}

Palavras-chave: Enfermeiro; CAPS; Esquizofrênico.

\begin{abstract}
Schizophrenia is a complex psychiatric disorder that begins early in an individual's life and presents several social and cognitive deficits characterized by distortions of thought, self-perception, delusions, hallucinations, psychosis, and external reality. The objective was to analyze the actions developed by nurses in the Psychosocial Care Centers CAPS aimed at the permanence of the schizophrenic patient in treatment. An integrative literature review study was carried out with several searches in the databases Virtual Health Library (VHL), BDENF, LILACS, and SCIELO, with the descriptors: "Nurse", "Schizophrenia", and "CAPS". A total of 305 articles were selected, and after the inclusion and exclusion criteria were applied, 31 articles were selected for reading. After reading, 7 articles were selected that met the research objective. The results of the sample showed that the existing actions are constantly being improved and new strategies are being developed to keep the patient in the treatment. It is concluded that the nurse stands out in the care provided to the user with schizophrenia in health services, due to the bond established and responsibility in the structuring of strategies for the promotion of mental health.
\end{abstract}

Keywords: Nurse; CAPS; Schizophrenic.

\section{Resumen}

La esquizofrenia es un trastorno psiquiátrico complejo que se inicia en las primeras etapas de la vida del individuo y presenta varios déficits sociales y cognitivos caracterizados por distorsiones del pensamiento, de la percepción del yo, delirios, alucinaciones, psicosis y de la realidad exterior. El objetivo es analizar las acciones de los enfermos en los Centros de Atención Psicosocial CAPS para la permanencia del paciente esquizofrénico en el tratamiento. Se 
realizó un estudio de revisión integradora de la literatura con varias búsquedas en las bases de datos Biblioteca Virtual en Salud (BVS), BDENF, LILACS, SCIELO, con los escritores: "Enfermeiro", "Esquizofrênia" y "CAPS". Se seleccionaron 305 artículos, a los que poco después se les aplicaron los criterios de inclusión y exclusión y se seleccionaron 31 artículos para su lectura. Tras la lectura se seleccionaron 07 artículos que cumplían el objetivo de la investigación. Los resultados de la encuesta evidencian que las acciones ya existentes están en constante mejora y se están desarrollando nuevas estrategias para lograr la permanencia del paciente en el tratamiento. Se concluye que las enfermeras se destacan en la atención a los usuarios con esquizofrenia en los servicios de salud, por el vínculo establecido y la responsabilidad en la estructuración de estrategias de promoción de la salud mental.

Palabras clave: Enfermera; CAPS; Esquizofrenia.

\section{Introdução}

A esquizofrenia é um transtorno psiquiátrico complexo de inicio cedo na vida do indivíduo e que apresenta diversos déficits sociais e cognitivos caracterizados por distorções do pensamento, da percepção de si, delírios, alucinações, psicose e da realidade externa. Os portadores do transtorno têm dificuldade em manter relações interpessoais normais e vínculo trabalhista, bem como atingir metas educacionais e ocupacionais. Levando em consideração alguns critérios etiológicos, podemos pressupor que a esquizofrenia existe a partir de um quadro endógeno e critérios evolutivos apontam a evolução retrógrada e invalidez psíquica do indivíduo (Costa, 2017). Atualmente, os pacientes com esquizofrenia são maioria nos leitos de hospitais psiquiátricos. Trata-se de uma doença bastante prevalente dentre as condições psiquiátricas. No Brasil aparecem cerca de 75.000 novos casos desse transtorno por ano, o que representa 50 casos para cada 100.000 habitantes (OMS, 2000).

É um transtorno de longa duração no qual o indivíduo experimenta períodos de crises e remissões que resultam em deterioração do funcionamento do doente e da família, causa diversos danos e perdas nas habilidades de todo grupo: diminuição da habilidade para cuidar de si mesmo, para trabalhar, para se relacionar individual e socialmente e para manter pensamentos completos (Giacon \& Galera, 2006).

Por se tratar de uma psicose, a esquizofrenia requer tratamento para toda vida, através de medicamentos e terapias psicossociais que ajudam a melhoras os sintomas da doença. O SUS oferece como ajuda o CAPS, que é o centro de apoio psicossocial, para pessoas com algum transtorno psiquiátrico, para que eles possam ser integrados na sociedade e dar apoio aos familiares que convivem com esse paciente. O tratamento medicamentoso é fundamental para controle da esquizofrenia, mas na avaliação dos pacientes, os prejuízos acarretados pelo tratamento medicamentoso podem ser tão intensos quanto os sintomas do transtorno. O tratamento recebido pelos portadores de esquizofrenia dificilmente se coloca à altura da complexidade do transtorno, que deve ser tratado em diversas frentes para que o paciente possa atingir uma boa qualidade de vida (Souza et. al, 2013).

Os serviços abertos surgiram em contraponto às instituições fechadas, tendo como objetivos "romper com a tendência carcerária da ideologia manicomial; manter os usuários o menor tempo possível na instituição; estimular a permanência dos usuários no núcleo familiar e social”. Entre esses projetos, encontram-se os Centros de Atenção Psicossocial (CAPS), que garante um "espaço de produção de novas práticas sociais para lidar com a loucura, o sofrimento psíquico, a experiência diversa; para a construção de novos conceitos, de novas formas de vida” (Soares \& Saeki, 2006).

Segundo os autores Cruz et al., (2016) é essencial conhecer as especificidades dos usuários para a identificação das demandas do serviço, avaliação das políticas de Saúde Mental e construção de propostas diferenciadas para o cuidado em tal contexto. Em concordância os autores Queiroz et al., (2016) afirma que há, muitas vezes, uma "CAPS-dependência" do usuário com o serviço, em que, mesmo em condição de alta, um grande número de usuários não se desliga do serviço, pois não criou vínculos com outros serviços e/ou pessoas no território, gerando a cronificação do usuário e do próprio CAPS.

As relações pessoais que os usuários constroem no serviço, tanto com profissionais de saúde como com outros usuários, faz com que se sintam à vontade para expressar opiniões, angústias e sentimentos. Isso fortalece o vínculo pessoal com os profissionais e, como consequência, faz com que os pacientes desenvolvam confiança nos profissionais, continuem no 
tratamento muitas vezes dificil e se sentem valorizados pelo serviço. Portanto, é importante que os serviços prestem abordagens apropriadas e que visam melhorar a adesão desta clientela devido ao distanciamento entre as motivações que levam o usuário a querer interromper o uso de medicamentos e a busca efetiva por tratamento (Grigolo, 2010).

Diante do exposto, o estudo tem como objetivo analisar, evidenciar e realizar a categorização na literatura todas as publicações que abordem a temática: As Ações de Enfermagem Voltadas a Permanência do Paciente Esquizofrênico Vinculados ao Centro de Atenção Psicossocial CAPS.

\section{Metodologia}

Trata-se de um estudo de revisão de literatura pelo método de revisão integrativa com abordagem qualitativa. A Revisão Integrativa configura-se, portanto, como um tipo de revisão da literatura que reúne achados de estudos desenvolvidos mediante diferentes metodologias, permitindo aos revisores sintetizar resultados sem ferir a filiação epistemológica dos estudos empíricos incluídos (Soares, 2014). De acordo com (Galvão e Pereira (2014) Os métodos para elaboração de revisões sistemáticas preveem: (1) elaboração da pergunta de pesquisa; (2) busca na literatura; (3) seleção dos artigos; (4) extração dos dados; (5) avaliação da qualidade metodológica;(6) síntese dos dados (metanálise); (7) avaliação da qualidade das evidências; e (8) redação e publicação dos resultados.

A pesquisa foi realizada durante o mês de Setembro de 2020, mediante busca online na Biblioteca Virtual em Saúde (BVS) nas bases de dados SCIELO, LILACS e BDENF com os seguintes descritores presentes no DECS: Esquizofrenia, Enfermagem, CAPS. Utilizaram-se como critério de inclusão os estudos que tinham entre seus descritores: Esquizofrenia, Enfermeiro, CAPS, textos completos em inglês, português e espanhol no período de 2015-2020 que abordassem a temática em estudo descritos na íntegra e publicados pelo menos nos últimos 05 anos. Como critério de exclusão, optou-se por não utilizar artigos que não correspondiam ao objeto de estudo, textos que se encontravam incompletos, artigos que não estivessem disponíveis na íntegra online, que não forneciam informações suficientes para a temática e aqueles que não possuíam os descritores determinados pelos pesquisadores.

Os dados foram coletados por meio de um formulário que continha as variáveis: Ano de publicação; Abordagem metodológica; Base de dados; Região geográfica; Periódico; Título do artigo; Autor e Contribuições do estudo. Estas variáveis foram dispostas em tabela e quadro, utilizando-se porcentagem simples, e posteriormente, foi realizada a criação de categorias que responderam aos objetivos do estudo, realizando a discussão de acordo com os resultados dos artigos trabalhados.

Essa revisão bibliográfica atesta-se nos aspectos éticos, zelando pela a legitimidade das informações e preservando a autoria dos artigos pesquisados, considerando as normas da Associação Brasileira de Normas Técnicas (ABNT) e do manual de normatização do Centro Universitário Santo Agostinho (UNIFSA).

Após a leitura crítica do material selecionado, foi realizado a análise detalhada do material bibliográfico para extração dos elementos essenciais dos estudos, para construção dos resultados e discussão da pesquisa realizando, portanto, a categorização.

Em consecutivo à análise dos estudos, os mesmos serão agrupados em um quadro contendo os autores, modalidade de estudo, abordagem metodológica e o tema dos artigos, e uma tabela com o ano, periódico de publicação e a frequência dos estudos por ano/periódico.

\section{Resultados e Discussão}

Durante a elaboração da pesquisa fez-se um levantamento das publicações relevantes ao tema, na base de dados foram encontrados 305 artigos relacionados aos descritores. Após este levantamento, utilizou como critério de escolha os estudos publicados nos anos de 2015 a 2020, com prevalência em idioma português, textos completos e que fossem referentes ao tema 
abordado. Após seleção realizada posteriormente a esta etapa, foram selecionados aqueles que se encontravam dentro dos critérios de inclusão propostos, ficando assim com 31 artigos, sendo excluídos da pesquisa 24 artigos por não se enquadrarem aos respectivos itens. Depois de realizada a leitura criteriosa dos artigos selecionados, para obtenção daqueles que possuíam relação com a temática, resultou-se em uma amostra final de 07 artigos refinados de acordo com os objetivos do estudo e distribuídos em diferentes periódicos.

A pesquisa foi realizada utilizando-se os DECS: Esquizofrenia, Enfermagem, CAPS, realizado com descritores individuais e cruzamentos duplos. A síntese dos descritores utilizados e de bases de dados e das referências selecionadas está descrita logo a seguir nos quadros:

Quadro 1 - Distribuição das referências obtida nas bases de dados Scielo, Lilacs e BDENF seguindo os descritores estabelecidos. Teresina, 2020.

\begin{tabular}{|c|c|c|c|}
\hline Base de dados & Descritores cruzados & Referências obtidas & $\begin{array}{c}\text { Referências } \\
\text { selecionadas }\end{array}$ \\
\hline Scielo & Enfermagem e CAPS & 18 & 1 \\
\hline Lilacs & $\begin{array}{c}\text { Esquizofrenia e } \\
\text { Enfermagem }\end{array}$ & 77 & 3 \\
\hline BDENF & Esquizofrenia e CAPS & 30 & 3 \\
\hline
\end{tabular}

Fonte: Pesquisa direta nas bases de dados, Teresina 2021.

De um modo em geral, os estudos selecionados retratam as ações desenvolvidas pelo enfermeiro ao paciente portador de esquizofrenia, ações de prevenção na atenção primária, abordagem na educação em saúde, o motivo da prevalência da doença, as políticas de saúde voltadas ao público, dentre outros. Foram selecionados sete trabalhos científicos que após a leitura na íntegra e foi realizada a distribuição em um quadro resumo com abordagem das seguintes variáveis: título, ano de publicação, autores e os principais resultados, conforme apresentado no Quadro 2. Os trabalhos foram enumerados de 1 a 7 para facilitar a análise e identificação. 
Quadro 2 - Distribuição das referências obtidas nas bases de dados, Scielo, Lilacs e BDENF seguindo os descritores determinados. Teresina, 2020.

\begin{tabular}{|c|c|c|c|c|}
\hline $\mathrm{N}^{\mathrm{o}}$ & TÍTULO & ANO & AUTORES & PRINCIPAIS RESULTADOS \\
\hline 1 & $\begin{array}{l}\text { A enfermagem e o } \\
\text { protagonismo do usuário no } \\
\text { CAPS: um estudo na } \\
\text { perspectiva construcionista }\end{array}$ & 2020 & $\begin{array}{l}\text { BOSSATO, } \\
\text { Hércules Rigoni } \\
\text { et al. }\end{array}$ & $\begin{array}{l}\text { O estudo aponta como a equipe de Enfermagem produz } \\
\text { ações para promover o protagonismo do usuário na } \\
\text { Reabilitação Psicossocial. Tais ações operam } \\
\text { principalmente por meio da comunicação criativa e por } \\
\text { intermédio do trabalho em rede. Tudo isso mediado por } \\
\text { uma equipe de Enfermagem que se percebe como antena } \\
\text { disponível para ações psicossociais e em negociação com } \\
\text { o território da pessoa assistida. }\end{array}$ \\
\hline 2 & $\begin{array}{c}\text { Perfil de pacientes com } \\
\text { transtornos mentais atendidos } \\
\text { no Centro De Atenção } \\
\text { Psicossocial do município de } \\
\text { Candeias }\end{array}$ & 2016 & $\begin{array}{l}\text { CRUZ LC, Carmo } \\
\text { DC, Sacramento } \\
\text { DMS, Almeida } \\
\text { MCP, Silveira } \\
\text { HF, Ribeiro } \\
\text { Júnior HL. }\end{array}$ & $\begin{array}{l}\text { Sabendo-se que há um predomínio de diagnósticos } \\
\text { confirmados de esquizofrenia em relação aos pacientes } \\
\text { assistidos no CAPS II de Candeias, para ambos os sexos, } \\
\text { vê-se que estes dados podem subsidiar um melhor } \\
\text { planejamento das ações e garantias de aperfeiçoamentos } \\
\text { na área da saúde pública mental do município acima } \\
\text { citado, especialmente em relação aos pacientes } \\
\text { esquizofrênicos, favorecendo a oferta de um serviço de } \\
\text { saúde pública de qualidade e excelência para a população } \\
\text { Candeense. }\end{array}$ \\
\hline 3 & $\begin{array}{l}\text { A percepção da enfermagem } \\
\text { sobre o relacionamento com } \\
\text { os cuidadores dos portadores } \\
\text { de Esquizofrenia: o olhar de } \\
\text { um serviço de referência }\end{array}$ & 2016 & $\begin{array}{l}\text { D’ASSUNÇÃO, } \\
\text { Cinthia Feliciano } \\
\text { et al. }\end{array}$ & $\begin{array}{l}\text { A implantação de grupos psicoeducativos, visitas } \\
\text { domiciliares, auxílio no enfrentamento da sobrecarga } \\
\text { familiar, estímulo à manutenção de outras atividades, } \\
\text { além do cuidado com o paciente, estão entre as } \\
\text { estratégias que podem ajudar a família a enfrentar as } \\
\text { mudanças impostas na história e na convivência } \\
\text { cotidiana com um membro acometido. }\end{array}$ \\
\hline 4 & $\begin{array}{l}\text { Produção do cuidado em } \\
\text { Saúde Mental: desafios para } \\
\text { além dos muros institucionais }\end{array}$ & 2016 & $\begin{array}{l}\text { FERREIRA, } \\
\text { Thayane Pereira } \\
\text { da Silva et al. }\end{array}$ & $\begin{array}{l}\text { Permitiu problematizar algumas dificuldades vivenciadas } \\
\text { por profissionais e gestores do serviço em se } \\
\text { territorializar no mundo vivo dos seus usuários. Ser um } \\
\text { serviço de base territorial deve significar muito além de } \\
\text { estar num determinado espaço geográfico, mas pertencer } \\
\text { a certo mundo, habitado por pessoas que produzem } \\
\text { modos de existir singulares. }\end{array}$ \\
\hline 5 & $\begin{array}{l}\text { Mudanças ocorridas na } \\
\text { prática profissional na área da } \\
\text { saúde mental frente à reforma } \\
\text { psiquiátrica brasileira na } \\
\text { visão da equipe de } \\
\quad \text { enfermagem }\end{array}$ & 2017 & $\begin{array}{l}\text { MAFTUM MA, } \\
\text { Silva AGS, Borba } \\
\text { LO, Brusamarello } \\
\text { T, Czarnobay J. }\end{array}$ & $\begin{array}{l}\text { Do enfermeiro em saúde mental é necessária uma } \\
\text { diferenciada atuação focada em novas propostas em que } \\
\text { haja uma transformação terapêutica das relações de } \\
\text { poder entre o profissional e o sujeito que gera a } \\
\text { necessidade de desenvolver habilidades e habilidades na } \\
\text { abordagem e no relacionamento com o paciente. }\end{array}$ \\
\hline 6 & $\begin{array}{l}\text { Cuidado de enfermagem na } \\
\text { Atenção Primária à Saúde ao } \\
\text { paciente com esquizofrenia }\end{array}$ & 2019 & $\begin{array}{l}\text { ZANETTI, Ana } \\
\text { Carolina } \\
\text { Guidorizzi et al. }\end{array}$ & $\begin{array}{l}\text { O cuidado de enfermagem ofertado aos usuários com } \\
\text { esquizofrenia na APS envolvem avaliação da } \\
\text { sintomatologia da esquizofrenia, a identificação dos } \\
\text { sinais de crise e recaídas, as avaliações de risco } \\
\text { para outras comorbidades, o acompanhamento do } \\
\text { tratamento clínico, a utilização de abordagem } \\
\text { centrada na pessoa com utilização de agenda, o } \\
\text { estímulo a autonomia, o registro de informações } \\
\text { acerca da assistência integral, atividades de inserção } \\
\text { social, orientações básicas de rotina e utilização de } \\
\text { estratégias de promoção de saúde com enfoque na } \\
\text { adoção de estilos de vida saudáveis. }\end{array}$ \\
\hline
\end{tabular}




\begin{tabular}{|c|c|c|c|c|}
\hline 7 & $\begin{array}{c}\text { Atuação e estratégias do } \\
\text { enfermeiro na ressocialização } \\
\text { do paciente esquizofrênico }\end{array}$ & 2016 & $\begin{array}{c}\text { ZÓCCOLI, Bruna } \\
\text { de Oliveira et al. }\end{array}$ & $\begin{array}{c}\text { Constatou-se a confirmação da fragilidade que existe na } \\
\text { relação interpessoal pelo tempo de dedicação na } \\
\text { terapêutica do profissional, as poucas altas do dispositivo } \\
\text { CAPS, os obstáculos que o profissional enfrenta para } \\
\text { manter essa relação próxima do paciente que muitas } \\
\text { vezes é o próprio causador de geração de estresse ou sua } \\
\text { família. }\end{array}$ \\
\hline
\end{tabular}

Fonte: Pesquisa direta em base de dados. Teresina-PI, 2021

A partir dos dados coletados para a análise observou-se que em 2016, houve um maior número de publicações, com $57,1 \%$, voltadas para abordagem do tema. Quanto à metodologia adotada nos artigos selecionados, foram descritivos de abordagem quantitativa, qualitativa e revisões integrativas com nenhum relato de caso. Através do que foi exposto nas publicações selecionadas, o estudo permitiu realizar-se a seguinte analise sobre a temática:

Quadro 3 - Análise sobre a temática.

\begin{tabular}{|l|l|}
\hline \multicolumn{1}{|c|}{ ARTIGOS } & \multicolumn{1}{|c|}{ EVIDÊNCIAS } \\
\hline $1,2,3,5$ & O tratamento do paciente esquizofrênico no CAPS \\
\hline $1,5,6,2$ & A atuação do enfermeiro no CAPS \\
\hline $4,7,2,5$ & $\begin{array}{l}\text { Razões do abandono do tratamento por parte do } \\
\text { paciente }\end{array}$ \\
\hline
\end{tabular}

Fonte: Pesquisa direta em base de dados. Teresina-PI, 2021

Após a leitura detalhada dos artigos selecionados observou-se a necessidade de categorizá-los segundo a similaridade de conteúdo. Dessa forma, foram elaboradas três categorias: O tratamento do Paciente Esquizofrênico no CAPS, A Atuação do Enfermeiro no CAPS e Razões do Abandono do Tratamento por Parte do Paciente.

\subsection{O tratamento do paciente esquizofrênico no CAPS}

A concepção de cotidiano do CAPS foi entendida como o lugar de existência de todas as pessoas, isto é, "o mundo de vida" e não, na visão do modo de viver, como uma ação mecânica e repetitiva, ou seja, não um contexto que determina a vida do homem, mas um local de movimento e de construção da vida (Zerbetto et al., 2011). Os CAPS são um modelo de espaço de criatividade, reconstrução de vida, novos saberes e práticas. Ao invés de excluir, disciplinar e medicar, atualmente adota-se um tratamento acolhedor, cuidadoso e que permite estabelecer pontes com a sociedade.

O cuidado passa a ter como premissa a defesa da vida e a percepção dos usuários como cidadãos pertencentes à comunidade. Os tratamentos ofertados pelo CAPS visam a integração e reintegração do paciente na comunidade e sua inserção familiar e social, com o propósito de colaborar para a reabilitação psicossocial do portador de transtorno mental. Para tanto, é necessário considerar as conexões que envolvem o sujeito buscando entendê-las como produtoras de vida (Ferreira et al., 2017). 
Por conseguinte, para atingir esses objetivos, faz-se necessário que o CAPS se transforme em um serviço inovador, onde haja produção de novas práticas sociais para lidar com o sofrimento psíquico, construindo novos conceitos e formas de vida e saúde. Acredita-se que as estratégias a serem pensadas e implementadas para garantir a qualidade da atenção e da assistência de enfermagem ao usuário que busca assistência no CAPS, bem como o fortalecimento do modelo de atenção psicossocial, envolvam a revisão dos currículos das escolas formadoras das diversas categorias e de novos cenários de formação profissional (Zerbetto et al., 2011).

O CAPS na vida do portador de esquizofrenia e seu familiar cuidador são sinônimos de reintegração social, onde a família tem participação integral, deste modo, a reabilitação pode ocorrer de vários modos respeitando os equipamentos de saúde mental existente, através de psicoterapia, terapia ocupacional, acompanhamento terapêutico, orientação familiar, abordagem psicossocial em instituições, grupos de autoajuda, ou seja, atividades que visam reinserir o sujeito na família e na sociedade, exercendo papéis anteriormente desenvolvidos.

\subsection{A atuação do enfermeiro no CAPS}

A enfermagem direciona suas atividades de forma diferenciada no tratamento dos doentes mentais, implicando atitude de respeito e dignidade para com enfermo, bem como ações voltadas às individualidades do sujeito e participação deste em seu processo de tratamento, valorizando e estimulando o autocuidado, a reinserção em grupos sociais e comunitários. As práticas terapêuticas que visam à reabilitação do indivíduo em sofrimento psíquico resgatam um trabalho com características coletivas que incluem a participação da família, da sociedade e do Centro de Atenção Psicossocial - CAPS, que amparam o doente e oferecem intervenção terapêutica (Zóccoli et al., 2016).

O trabalho da Enfermagem no CAPS é primordial uma vez que permite a escuta, o acolhimento, o vínculo, a corresponsabilização e apoio emocional, nessa articulação, a Enfermagem atua em tempo integral nos cuidados cotidianos, sendo um dos membros da equipe de profissional de saúde que trabalha 24 horas com o usuário dentro das modalidades dos CAPS que funcionam em atenção contínua (BOSSATO et al., 2020).

Assim, a enfermagem desempenha um papel importante no processo de cuidado e reabilitação dos sujeitos acometidos pela esquizofrenia e seus familiares, a assistência tem como objetivo promover o cuidado integral em saúde mental para esta clientela abrangendo as distintas fases do transtorno mental, bem como diferentes níveis de atenção à saúde. São pautadas pela singularidade, porém ainda necessitam efetivar-se como terapêuticas e com uma abordagem no cuidado psicossocial e não apenas em planos e procedimentos prescritivos que desvalorizam a autonomia da pessoa que necessita do serviço de saúde mental (Zanetti et al., 2019)

Portanto, o enfermeiro passou a ocupar uma posição de agente terapêutico, não mais realizando apenas ações voltadas para o cuidado, mas também buscando compreender a relação entre sofrimento psicológico, político e social do paciente (Pintor et al., 2018).

Essa mudança de foco na assistência ao portador de transtorno mental, levou também o enfermeiro à necessidade de fazer adaptações na sua prática profissional para a adequação a esse novo contexto, pois, mesmo ainda sendo necessária a assistência em serviços fechados, ou seja, a internação por período integral para os casos de maior gravidade, os mesmos passaram também a funcionar dentro de uma proposta reabilitadora de transformação seguindo algumas diretrizes específicas, pautadas no entendimento do portador de transtorno mental como um ser humano e como um cidadão (Lima et al., 2010).

Desta modo, é necessário que haja uma atuação diferenciada exigindo que o profissional enfermeiro seja menos focado no diagnóstico e mais comprometido com o atendimento humanizado, visando novas propostas que favoreçam transformação terapêutica das relações de poder entre o profissional e o sujeito que gera a necessidade de desenvolver habilidades na abordagem e no relacionamento com o paciente (Maftum et al., 2017). 
Desta forma, o enfermeiro se destaca como articuladores do cuidado prestado ao usuário com esquizofrenia nos serviços de saúde, devido o vínculo estabelecido e responsabilidade na estruturação de estratégias de promoção em saúde mental. Estes profissionais devem priorizar o desenvolvimento de atividades individuais ou grupais, promover espaços de treinamento em habilidades de vida e gerenciar essas habilidades com o objetivo de oferecer alívio das emoções, solução de problemas, tomada de decisão, comunicação efetiva, empatia, autoestima, entre outros aspectos (Zanetti et al., 2019).

Na percepção dos enfermeiros, fica cada vez mais evidente que humanizar em saúde mental significa acolher, ouvir e com isso dar respostas positivas às necessidades individuais e coletivas das pessoas cuidadas. Significa cuidar das pessoas com responsabilidade, compromisso e ética, ajudando-as a vencer suas limitações. Reconhecem ainda que vários são os desafios a serem enfrentados no trabalho realizado no CASP que decorrem dos problemas práticos que vão desde as fragilidades nas competências e habilidades para lidar com o novo enfoque da assistência em saúde mental até a falta de recursos materiais e a superposição de atividades decorrentes das demandas dos serviços (Oliveira et al., 2015).

A participação do enfermeiro em conjunto com as práticas de reinserção social do CAPS através de relações interpessoais tanto com o paciente como o cuidador pode tornar-se uma ferramenta adicional à vida adequada e digna merecida dos indivíduos esquizofrênicos, favorecendo uma maior adesão ao tratamento, diminuindo assim as internações em hospitais psiquiátricos. (Soratto et al., 2014)

O relacionamento interpessoal entre o profissional e o cuidador é compartilhado por meio de sentimentos e emoções decorrentes da vivência com o portador de esquizofrenia. A busca por informações é o meio que a família utiliza para entender os acontecimentos e ressignificar seus sentimentos. Ao fornecer informações no tempo curto, a enfermagem pode estabelecer um relacionamento que exerce influência positiva nos tempos curto e vivido da família e poderá ter como uma das consequências melhorias na assistência ao portador. As estratégias como a empatia, o diálogo, a reunião de família e o encaminhamento para outros profissionais surgem como possibilidades de atender às necessidades expressas por meio de sentimentos. (D’Assunção et al., 2016).

Os profissionais destacam o seu envolvimento pessoal como uma marca do cuidado ao paciente esquizofrênico, trazendo sua interação como instrumento sem apropriação teórica do método da relação interpessoal terapêutica, uma assistência que requer um envolvimento pessoal e emocional junto ao paciente esquizofrênico que é inevitável no processo do cuidar. Os profissionais de enfermagem afirmam que o relacionamento interpessoal terapêutico é uma forma de abordagem que os profissionais da saúde podem utilizar, inclusive os da enfermagem. (Lima et al., 2013).

\subsection{Razões do abandono do tratamento por parte do paciente}

Até a década de 1980, a terapêutica para portadores de transtorno mental centrava-se no modelo hospitalocêntrico que se restringia a ofertar tratamento moral e medicamentoso. Além do uso de medicações e dopagem, o tratamento moral isolava e vinculava o indivíduo continuamente com a finalidade de regular e "normatizar" sua vida. Quando necessário, eram utilizados meios mais severos como colete de força, privações de visitas, de comida e por vezes os pacientes tinham sequer direito a ingerir água. Deste modo, as condutas não encorajavam o paciente ao prosseguimento do tratamento já que, na visão deles a assistência oferecida provocava repercussões negativas, sensação de punição e medo (Moll et al., 2012).

Outro fato relevante sobre os motivos para o abandono do tratamento por parte desses pacientes são os incômodos ocasionados pela farmacoterapia, pois, os medicamentos de primeira escolha para o tratamento da esquizofrenia são os antipsicóticos, no qual destacam-se como efeitos colaterais, os efeitos extrapiramidais que relaciona-se ao controle da postura e tônus muscular, agranulocitose que pode desencadear ulcerações no intestino, mucosas e garganta, ganho de peso, alterações metabólicas, sedação, fadiga, hipotensão ortostática, boca seca, sialorreia, náusea e vômito, redução do limiar para crises convulsivas, redução da libido, impotência sexual, amenorreia e ginecomastia (Verdana et al., 2013). Assim, a não adesão à 
terapia medicamentosa está associada a a exacerbação dos sintomas, piora do prognóstico, readmissões, altos custos, ajustes desnecessários em prescrições e refratariedade ao medicamento.

Mesmo com efeitos adversos e dificuldade de aceitação, a terapia medicamentosa é essencial para controlar os sintomas caracteristicos doença, especialmente quando combinados com outras modalidades terapêuticas, então, a adesão ao tratamento farmacológico e a segurança do paciente são desafios importantes na prática da saúde. É importante investigar o paciente e reconhecer as dificuldades relacionadas ao monitoramento da terapia medicamentosa, a fim de implementar ações para otimizar a aceitação e contribuir para a prevenção de lesões resultantes do uso inadequado dos fármacos (Miasso et al., 2015).

Reconhece-se portanto, que muitos desses fatores associados à não adesão estão intercambiados, como a persistência de sintomas psicóticos entre as crises, maior prevalência de tentativas de suicídio, maior uso dos serviços de emergência, maior número de reinternações e maiores gastos com a saúde, gerando prejuízos não somente para o indivíduo em questão, mas também para o sistema de saúde como um todo, já que promove o saturamento da rede e custos desnecessários com os serviços (Silva et al., 2012).

Verificou-se por meio de pesquisas que a maioria dos pacientes abandonou a terapêutica no período de 1 a 6 meses, ou seja, no início do tratamento, já nos demais períodos superiores a 6 meses e inferiores a 48 meses, os resultados demonstraram uma constante. Diante disso torna-se significativo a pesquisa para explorar as melhores estratégias para intervir nas dificuldades, conflitos e na necessidade de conhecimento entre pessoas com esquizofrenia para promover adesão ao tratamento e segurança do paciente (Moll et al., 2012).

\section{Conclusão}

Com a pesquisa observou-se que a Enfermagem exerce funções de extrema importância na reabilitação de pacientes com transtorno esquizofrênico dentro dos CAPS, de forma que devem sempre estimular a reinserção destes no meio social.

Deste modo é necessário que haja uma parceria entre a família, os profissionais, alinhado juntamente com tratamentos psicológicos, psiquiátricos, terapia ocupacional e ainda medicamentoso, estimulando que o paciente se sinta seguro e permaneça no tratamento até estar apto para se reintegrar socialmente. Essas ações e novas intervenções da enfermagem podem facilitar a vivencia de uma pessoa numa situação de saúde/doença ajudando na redução dos sintomas.

Com a pesquisa observou que o enfermeiro se destaca no cuidado prestado ao usuário com esquizofrenia nos serviços de saúde, devido o vínculo estabelecido e responsabilidade na estruturação de estratégias de promoção em saúde mental. Em destaque a necessidade de estratégias especificas para cada paciente, realizando e montando um programa que seja personalizado e adaptado para este indivíduo.

Assim, sugere-se que haja mais estudos abordando a temática com o intuito de buscar mais conhecimento e embasamento científico, mais ações e inovações para que a equipe de Enfermagem, principalmente enfermeiros, tornem-se cada vez mais habilitados e capazes de cooperar para a reabilitação de pacientes esquizofrênicos.

Como perspectiva futura, o presente estudo busca servir de base para futuras novas pesquisas sobre a temática, instigar a curiosidade para elaboração de novos trabalhos com a temática, sugerindo que proponham novas estratégias que auxiliem o usuário diante as necessidades que possam passar durante o período do tratamento, o que faz pensar em atividades de educação continuada voltada à estes como, por exemplo, referentes ao cuidado com o paciente, formas de facilitar esse processo, ou novas estratégias que auxiliem na melhoria da qualidade da assistência do enfermeiro para esse grupo em estudo, afim de promover a permanência do paciente esquizofrênico no tratamento, melhorando a adesão ao mesmo, desde as primeiras semanas, uma vez que a assistência ao paciente esquizofrênico ainda é recoberta por muitos desafios. 


\section{Referências}

Bossato, H. R., Oliveira, R. M. P. D., Dutra, V. F. D., \& Loyola, C. M. D. (2021). A enfermagem e o protagonismo do usuário no CAPS: um estudo na perspectiva construcionista. Revista Gaúcha de Enfermagem, 42(SPE), e20200082.

Costa, R. F. (2018). Physical activity, lifestyle-related factors and psychological determinants among patients with schizophrenia.267, 382-393

D'Assunção, C. F., dos Santos, A. L. D., Lino, F. A., \& Silveira, E. A. A. (2016). A percepção da enfermagem sobre o relacionamento com os cuidadores dos portadores de Esquizofrenia: o olhar de um serviço de referência. Revista de Enfermagem do Centro-Oeste Mineiro. 6(1) $2034-2051$.

Nascimento P. R. P., Aguiar, A. S. C., Cândido, D. A., Monteiro, A. R. M., Almeida, P. C., Roscoche, K. G. C., \& Reis, P. A. M. (2019). Análise do perfil de usuários atendidos em um Centro de Atenção Psicossocial/Analysis of the profile of users assisted in a psychosocial care center/Análisis del perfil de usuarios atendidos en un centro de atención psicosocial. Journal Health Npeps. 4(1), 132-143.

Ferreira, T. P. D. S., Sampaio, J., Souza, A. C. D. N., Oliveira, D. L. D., \& Gomes, L. B. (2017). Care production in Mental Health: the challenges beyond institutional walls. Interface-Comunicação, Saúde, Educação, 21(61), 373-384.

Ferreira, T. P. D. S., Sampaio, J., Souza, A. C. D. N., Oliveira, D. L. D., \& Gomes, L. B. (2016). Produção do cuidado em Saúde Mental: desafios para além dos muros institucionais. Interface-Comunicação, Saúde, Educação, 21, 373-384.

Galvão, T. F., \& Pereira, M. G. (2014). Revisões sistemáticas da literatura: passos para sua elaboração. Epidemiologia e Serviços de Saúde, $23,183-184$.

Giacon, B. C. C., \& Galera, S. A. F. (2006). Primeiro episódio da esquizofrenia e assistência de enfermagem. Revista da Escola de Enfermagem da USP, 40(2), 286-291.

Grigolo, T. M. (2010). O Caps me deu voz, me deu escuta: um estudo das dimensões da clínica nos Centros de Atenção Psicossocial na perspectiva de trabalhadores e usuários. 283f, 1-283.

Lima, D. U. D., Garcia, A. P. R. F., \& Toledo, V. P. (2013). Compreendendo a equipe de enfermagem na assistência ao paciente esquizofrênico. Revista Rene; 14(3), 503-11.

Lima, R. V. M. D., Pedrão, L. J., Gonçalves, J. G., \& Luis, M. A. V. (2010). Papéis, conflitos e gratificações do enfermeiro de serviços abertos de assistência psiquiátrica. Revista Eletrônica de Enfermagem, 12(2), 348-53.

Maftum, M. A., Pagliace, Â. G. D. S., Borba, L. D. O., Brusamarello, T., \& Czarnobay, J. (2017). Changes in professional practice in the mental health area against brazilian psychiatric reform in the vision of the nursing team. Revista de Pesquisa: Cuidado é Fundamental Online, 9(2), $309-314$.

Miasso, A. I., Miamoto, C. S., do Carmo Mercedes, B. P., \& Vedana, K. G. G. (2015). Adesão, conhecimento e dificuldades relacionados ao tratamento farmacológico entre pessoas com esquizofrenia. Revista Eletrônica de Enfermagem, 17(2), 186-95.

MOLL, M. F., Oliveira, K. J. A., Emerson dos Reis, D. I. A. S., \& VENTURA, C. A. A. (2012). O abandono ao tratamento entre pacientes assistidos em um Centro de Atenção Psicossocial. Journal of Nursing and Health, 2(1), 18-27.

Oliveira, L. C. D., Silva, R. A. R. D., Medeiros, M. N. D., Queiroz, J. C. D., \& Guimarães, J. (2015). Humanized care: discovering the possibilities in the practice of nursing in mental health. Revista de Pesquisa: Cuidado é Fundamental Online, 7(1), 1774-1782.

Organização Mundial da Saúde. (2000). Classificação estatística internacional de doenças e problemas relacionados à saúde: CID-10.

Pintor, L. A., Toledo, V. P., \& Garcia, A. P. R. F. (2018). Nursing care based on the perspective of the subject of the unconscious and its contribution to the Singular Therapeutic Project. SMAD. Revista eletrônica saúde mental álcool e drogas, 14(1), 20-27.

Silva Junior, A. G. D., \& Mascarenhas, M. T. M. (2004). Avaliação da atenção básica em saúde sob a ótica da integralidade: aspectos conceituais e metodológicos. In Cuidado: as fronteiras da integralidade (pp. 241-257).

Silva, T. F. C. D., Lovisi, G. M., Verdolin, L. D., \& Cavalcanti, M. T. (2012). Adesão ao tratamento medicamentoso em pacientes do espectro esquizofrênico: uma revisão sistemática da literatura. Jornal Brasileiro de Psiquiatria, 61(4), 242-251.

Soares, C. B., Hoga, L. A. K., Peduzzi, M., Sangaleti, C., Yonekura, T., \& Silva, D. R. A. D. (2014). Revisão integrativa: conceitos e métodos utilizados na enfermagem. Revista da Escola de Enfermagem da USP, 48(2), 335-345.

Soares, S. R. R., \& Toyoko, S. (2006). O centro de atenção psicossocial sob a ótica dos usuários. Revista Latino-Americana de Enfermagem, 14(6), 923-929.

Soratto, M. T. (2014). O CAPS na vida do portador de esquizofrenia. Enfermagem Brasil, 13(3), 141-146.

Souza, J. D., Almeida, L. Y. D., Veloso, T. M. C., Barbosa, S. P., \& Vedana, K. G. G. (2013). Estratégia de Saúde da Família: recursos comunitários na atenção à saúde mental. Acta Paulista de Enfermagem, 26(6), 594-600.

Vedana, K. G. G., Cirineu, C. T., Zanetti, A. C. G., \& Miasso, A. I. (2013). Agindo em busca de alívio: enfrentamento da esquizofrenia e dos incômodos ocasionados pelo tratamento medicamentoso/Acting for relief: coping with schizophrenia and nuisances caused by drug treatment. Ciência, Cuidado $e$ Saúde, 12(2), 365-374.

Zanetti, A. C. G., Rossi, M., Vedana, K. G. G., Gherardi-Donato, E. C., \& Gouveia, R. I. (2019). Cuidado de enfermagem na Atenção Primária à Saúde ao paciente com esquizofrenia. Saúde \& Transformação Social/Health \& Social Change, 10(1/2/3), 201-208. 
Research, Society and Development, v. 10, n. 6, e13010615717, 2021

(CC BY 4.0) | ISSN 2525-3409 | DOI: http://dx.doi.org/10.33448/rsd-v10i6.15717

Zerbetto, S. R., Efigênio, E. B., dos Santos, N. L. N., \& Martins, S. C. (2011). O trabalho em um Centro de Atenção Psicossocial: dificuldades e facilidades da equipe de enfermagem. Revista Eletrônica de Enfermagem, 13(1), 99-109.

Zóccoli, B. D. O., Prado, F., Roldão, M. D. S., \& Pereira, V. P. (2016). Atuação e estratégias do enfermeiro na ressocialização do paciente esquizofrênico. Revista Eletrônica Estácio Saúde, 5(1), 48-60. 